\title{
Adolescents, THC, and the risk of psychosis
}

Elohor Sandra Otite, MD, Amrita Solanky, MD, and Stacy Doumas, MD

Editor's note: Readers' Forum is a department for correspondence from readers that is not in response to articles published in Current Psychiatry. All submissions to Readers' Forum undergo peer review and are subject to editing for length and style. For more information, contact letters@currentpsychiatry.com.

S ince the recent legalization and decriminalization of cannabis (marijuana) use throughout the United States, adolescents' access to, and use of, cannabis has increased. ${ }^{1}$ Cannabis products have been marketed in ways that attract adolescents, such as edible gummies, cookies, and hard candies, as well as by vaping. ${ }^{1}$ The adolescent years are a delicate period of development during which individuals are prone to psychiatric illness, including depression, anxiety, and psychosis. ${ }^{2,3}$ Here we discuss the relationship between adolescent cannabis use and the development of psychosis.

\section{How cannabis can affect the adolescent brain}

The 2 main psychotropic substances found within the cannabis plant are tetrahydrocannabinol (THC) and cannabidiol (CBD). ${ }^{1,4}$ Endocannabinoids are fatty acid derivatives produced in the brain that bind to cannabinoid (CB) receptors found in the brain and the peripheral nervous system. ${ }^{1,4}$

During adolescence, neurodevelopment and neurochemical balances are evolving, and it's during this period that the bulk of prefrontal pruning occurs, especially in the glutamatergic and gamma aminobutyric acidergic (GABAergic) neural pathways. ${ }^{5}$
THC affects the CB1 receptors by downregulating the neuron receptors, which then alters the maturation of the prefrontal cortical GABAergic neurons. Also, THC affects the upregulation of the microglia located on the $\mathrm{CB} 2$ receptors, thereby altering synaptic pruning even further., ${ }^{2,5}$

All of these changes can cause brain insults that can contribute to the precipitation of psychotic decompensation in adolescents who ingest products that contain THC. In addition, consuming THC might hasten the progression of disorder in adolescents who are genetically predisposed to psychotic disorders. However, existing studies must be interpreted with caution because there are other contributing risk factors for psychosis, such as social isolation, that can alter dopamine signaling as well as oligodendrocyte maturation, which can affect myelination in the prefrontal area of the evolving brain. Factors such as increased academic demand can alter the release of cortisol, which in turn affects the dopamine response as well as the structure of the hippocampus as it responds to cortisol. With all of these contributing factors, it is difficult to attribute psychosis in adolescents solely to the use of THC. ${ }^{5}$

\section{How to discuss cannabis use with adolescents}

Clinicians should engage in open-ended therapeutic conversations about cannabis use with their adolescent patients, including the various types of cannabis and methods of use (ingestion vs inhalation, etc). Educate patients about the acute and long-term
Dr. Otite is a Research Volunteer, Department of Psychiatry, Jersey Shore University Medical Center, Neptune, New Jersey. Dr. Solanky is a PGY-1 Psychiatry Resident, Department of Psychiatry, Ocean Medical University Center, Brick, New Jersey. Dr. Doumas is Chief, Child and Adolescent Psychiatry; Residency Program Director; and Vice Chair of Education and Research, Department of Psychiatry, Jersey Shore University Medical Center, Neptune, New Jersey; and Vice Chair, Department of Psychiatry, Hackensack Meridian School of Medicine, Nutley, New Jersey.

Disclosures

The authors report no financial relationships with any companies whose products are mentioned in this article, or with manufacturers of competing products.

doi: 10.12788/cp.0197

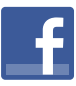

Discuss this article at www.facebook.com/ MDedgePsychiatry 


\section{Clinical Point}

Because there are many contributing risk factors, it is difficult to attribute psychosis in an adolescent solely to the use of THC effects of THC use, including an increased risk of depression, schizophrenia, and substance abuse in adulthood.

For a patient who has experienced a psychotic episode, early intervention has proven to result in greater treatment response and functional improvement because it reduces brain exposure to neurotoxic effects in adolescents. ${ }^{3}$ Access to community resources such as school counselors can help to create coping strategies and enhance family support, which can optimize treatment outcomes and medication adherence, all of which will minimize the likelihood of another psychotic episode. Kelleher et $\mathrm{al}^{6}$ found an increased risk of suicidal behavior after a psychotic experience from any cause in adolescents and young adults, and thereby recommended that clinicians conduct continuous assessment of suicidal ideation in such patients.

\section{References}

1. US Food \& Drug Administration. 5 Things to know about delta-8 tetrahydrocannabinol - delta- 8 THC. Updated September 14, 2021. Accessed November 3, 2021. https:// www.fda.gov/consumers/consumer-updates/5-thingsknow-about-delta-8-tetrahy drocannabinol-delta-8-thc

2. Patel PK, Leathem LD, Currin DL, et al. Adolescent neurodevelopment and vulnerability to psychosis. Biol Psychiatry. 2021;89(2):184-193. doi: 10.1016/j. biopsych.2020.06.028

3. Kane JM, Robinson DG, Schooler NR, et al. Comprehensive versus usual community care for first-episode psychosis: 2-year outcomes from the NIMH RAISE early treatment program. Am J Psychiatry. 2016;173(4):362-372. doi: 10.1176/ appi.ajp.2015.15050632

4. Mastrangelo M. Clinical approach to neurodegenerative disorders in childhood: an updated overview. Acta Neurol Belg. 2019;119(4):511-521. doi: 10.1007/s13760-019-01160-0

5. Sewell RA, Ranganathan M, D'Souza DC. Cannabinoids and psychosis. Int Rev Psychiatry. 2009;21(2):152-162. doi: 10.1080/09540260902782802

6. Kelleher I, Cederlöf M, Lichtenstein P. Psychotic experiences as a predictor of the natural course of suicidal ideation: a Swedish cohort study. World Psychiatry. 2014;13(2):184-188. doi: $10.1002 /$ wps.20131 